\title{
Ergonomic design: experimental studies of multi-task vehicle
}

\author{
Eyal Byran ${ }^{1}$, Issachar Gilad ${ }^{2}$, Rivka Oxman ${ }^{1}$ \\ ${ }^{1}$ Faculty of Architecture and Town planning, Technion-Israel Institute of Technology, Haifa, Israel \\ ${ }^{2}$ Faculty of Industrial Engineering and Management, Technion-Israel Institute of Technology,Haifa, Israel
}

\section{Email address:}

byrane@gmail.com (E. Byran)

\section{To cite this article:}

Eyal Byran, Issachar Gilad, Rivka Oxman. Ergonomic Design: Experimental Studies of Multi-Task Vehicle. Agriculture, Forestry and Fisheries .Vol. 2, No. 1, 2013, pp. 23-32. doi: 10.11648/j.aff.20130201.13

\begin{abstract}
The design of off-road vehicles is complicated. Particularly when the driving, is accompanied with another task, simultaneously (e.g. plowing). Such a complex environment may lead to human error and end up with an accident. In Australia, 20-25 percent of vehicle accidents' casualties are from off-road accidents. The literature suggests three combined and important aspects, in vehicle design: vision control, anthropometrical fitting and biomechanical considerations. No study is found which supports an off-road vehicle design from all three aspects together. In addition, the extensive use of computer aided design (CAD) in the vehicles' design stages, produce only qualitative results. This study presents a pioneering experimental ergonomic research, of an off-road cabin design. The research inquires the three aspects,combined, generating quantitative results. These results produce a better fitted design to the operator and his operational needs, which help the engineer to design a cabinthat may lead to less strenuous sitting postures and fatigue.
\end{abstract}

Keywords: Ergonomics; Vehicle; Automotive; Biomechanics; Tractors, Off road vehicle; Cabin design; Safety; Human error

\section{Introduction}

This chapter presents the importance of ergonomics in workplace design, and its contribution in increasing the efficiency and safety of the worker by minimizing the exposure to work related hazards. Several relevant researches are discussed regarding the necessity of ergonomic in vehicle design and especially in off-road vehicles.

A good working posture was first characterized in the beginning of the eighteenth century. It was the first time that the importance of avoiding unnatural postures of the human body during work performance, was brought into discussion. Since then, basic guidelines and recommendations are published in the scientific literature regarding 'good' or 'healthy' workplace. The importance of ergonomic thinking in design is described, for example, in the work of [21] for adopting ergonomic set of rules in the design of hand tools. Later, [2], showed the importance of fitting every design to the human. In his work, [14] adds that the ergonomic thinking does not apply only to the early design stages, but to all product life cycle, through various assessments. This improves the design even more to match the human requirements.
In architecture, on the contrary, ergonomic design is lacking, as suggested by [6]. He suggests that designers should take in mind the fact that spaces should be designed and fitted for the usage of living and functioning humans, and not just become pure art. Regarding this last issue [1], suggest the need of comprehensive methodology for ergonomic design of all architectural indoor and outdoor spaces. Spaces, that can be described as surroundings or environments that humans must function inside them.

As such, a cabin of a conventional vehicle can be described in terms of architectural space. This confined space can be a very complex, containing many additional systems, which may distract the driver from his main task, rather than support a safe vehicle operation. Although the researchers have not reached a solid single definition about mental workload, they all agree on one point; mental workload can be described as a relation between the demand(s) resulting from the various tasks to be performed, on the human operator, and his ability to fulfill these demands satisfactory. These demands, as stated by [30], are often described as multidimensional, as they involve tasks, operator and system demands together with other factors. Therefore, the importance of well fitted architectural space 
to the human driver's dimensions and needs is very crucial. In addition, mental workload level increases with the time passing. Since multi-task vehicle drivers spend inside the cabin many hours, sometimes even most of the day or night, this may increase the level of the mental workload, possibly leading to human error. Similar conclusion was suggested by [18]. Their conclusion was that the vehicle design should be human oriented in order to maximize comfort and in addition to be best fitted to the human dimensions and follow all cognitive demands in order to make it possible for the human driver to perform the driving task at his best and with maximum safety and minimal risks of human error.

The large number of published articles found in the scientific literature, suggests that the vehicle design is found to be an intriguing and indeed a complicated environment. Off-road vehicles that involve more than just driving ahead (like a tractor that besides driving ahead also plows the ground) are considered even more complicated in terms of design environment than conventional vehicles. These vehicles demand from the operator to perform multiple tasks simultaneously, hence these vehicles are called multi-task vehicles.

When defining human oriented vehicle design, most researches agree that the need for visual ability of the road ahead together with the need for manual operation by the driver in a seated position, demand a well fitted environment or a 'Good working posture'. The term was introduced and defined through basic guidelines first by [7]. He suggested that a certain posture is adopted, because it is the most appropriate for the performance of a specific task. The factors that associate with an acquired posture at a performed task are: visual needs, reaching and manipulative needs, postural and biomechanical loads. In addition, to the above mentioned factors, the resulting posture, is affected by any constrains and obstructions imposed by the specified space which limit the ability to see, reach, or exert force. The visual aspects can be affected by additional environment factors such as vehicle vibration, constrains and obstructions imposed by the specified cabin or vehicle geometry.

The relevance and importance of ergonomic thinking inside the vehicle's cabin design is very clear. The cabin design is found in the scientific literature very challenging from many angles and aspects, and many studies and design recommendations have been published regarding the ergonomic suitability of the cabin and it's various components' design. The research conducted by [11], investigated tractor accidents in Australia between the years 1989 - 92. Out of 87 tractors accidents, the average percent of fatalities per year was 24 (21 fatalities); or approximately two fatalities every month. $78.2 \%$ of the accidents involved vehicle accidents; most of them were tractor rollovers $(55.2 \%) .21 .8 \%$ of the accidents involved bystanders' accidents, most of them being hit by a tractor $(37.9 \%)$. The most common activities being performed at the time of the accident were working with crops $(25.3 \%)$, transport for work purposes (19.5\%) and clearing land (10.3\%). This information intensify the assumption that the drivers' field-of-view was partially blocked, leading to human error that ended in accidents and casualties.

In 1995 the American National Aeronautics and Space Administration (NASA) published a complete manual for design guidelines. This manual includes chapters on ergonomic design guidelines for their aircrafts and shuttles cockpits, [22]. Later, in 1999 the US Military published his own manual [20], which includes ergonomic guidelines for vehicle cabins (transportation and armored vehicles).

In 2000, during the Human Factors and Ergonomics Society (HFES) congress, the issue of the driver's workplace was discussed. The Institute of Industrial Engineering and Ergonomics in Aachen, University of Technology in Germany, was dealing with a way to evaluate the driver's workplace design in vehicles, [25]. Most of the vehicles that were evaluated were private vehicles, only two of them were public transport vehicles and one was an agricultural vehicle (a combine). The various evaluations led the researches to acquiring a set of methods. They state in their article that the methods that were used during the evaluation possesses can be divided into three categories: a) Geometric fitting - by analyzing the static posture of the human operator inside a given cabin; b) measurement of body angles during a long period of time using video recordings analysis; and c) distribution of eye movement during driving a vehicle analysis for identifying general viewing sectors. This is the only paper in the scientific literature that was found that deals with a way to evaluate the driver's workplace from several aspects, and by using quantifying methods. These aspects were analyzed one at a time rather than combined and depended on one another.

In 2006, a European multidisciplinary team, assembled from several European countries and professions was gathered. On their agenda was the writing of a guideline manual for agriculture, forest and off-road vehicles. They generated a detailed checklist document for ergonomic and safe usage of field vehicles that graded the vehicle design and its components from different aspects; one of them was the ergonomic aspect. This document is called 'Guidelines for Forest Machines European Ergonomic and Safety, Ergowood, 2006', [10]. Following this checklist lets the user to make a basic evaluation of how well the vehicle follows safety and ergonomic guidelines and regulations. This document does not evaluate the actual fitting of the cabin to the human dimensions (e.g. no anthropometrical fitting) and does not support any visual evaluation.

Although these examples are a giant step in combining several ergonomic aspects toward a better and efficient workplace, they do not provide the designer with whole quantitative information of how well the geometry layout supports the safety and comfort of the driver, and they do not present the amount of actual clear visual field-of-view for each task.

Today when all vehicles are planned and designed by computer using state-of-the -art Computer Aided Design 
(CAD) programs with ergonomic add-ons and advanced simulation abilities, people may think that the cabin design would be better ergonomically designed and fitted to the human operator. In reality the picture is the opposite. [5]suggests, that only ten percent of the engineers that are actually involved in the vehicle design process, have some (or even less) ergonomic knowledge.

To conclude, the literature suggests insufficient information regarding a way to approach a multi-task or off-road vehicle design, based on the three ergonomic aspects mentioned above that were found to be fundamental. Many of the articles found in the literature are dealing with only one aspect, such as vision or manual controlling, omitting the other aspects. Therefore, they do not provide the designer in the early planning stages, or the driver of the vehicle with the sufficient guidelines, or a way to design/adjust the components inside the cabin for best fitting (i.e. larger clear field-of-vision and better sitting posture).

The main objective of the research is to describe an experimental new way to approach the design of a multi-task off-road cabin, with ergonomic capabilities, in which, the driver has more than just steering tasks to accomplish. The methodology that will be used, will explore the cabin design in three different ergonomic aspects simultaneously: vision - the actual visual abilities of the driver including the binocular vision of 120 degrees (horizontally); anthropometry - the measurement of the human driver; and biomechanics - the study of the structure and function of the human driver by methods of mechanics.

\section{Methodology}

The investigation is based on three separate studies that depend on each other: (1) clear field-of-view study, (2) anthropometric study (hand and foot reach envelopes), and (3) driver's upper body (neck, shoulders and lower back) biomechanical study. The clear field-of-view study checks the driver's capabilities to view the road ahead and to his near sides without any interference. The anthropometric study checks the locations of all control in relation to the driver's dimension. The biomechanical study, checks the loads on the driver's upper body as a result of his sitting posture. The results from each study are unequivocal and quantitative. These three aspects are equally important, as they implicate on each other. When these aspects are addressed together, the results contribute to the creation of an ergonomic efficient workplace's design that fits the operator and his needs with safety and health at the top priority. The output of these studies would yield design geometry that may suggest better comfort for the driver, thus, increase the driver overall effectiveness. It may also lead to less fatigue and strenuous sitting postures, more vigilance driving and controlling of the vehicle; assuming, this will help reduce road accidents that occur out of human errors driven from fatigue. As mentioned before, such a way for cabin design of a multi-task vehicle, driven by ergonomic considerations, does not exist in the scientific literature.
The following approach is suggested:

Vehicle parameters, dimensions and geometry study. Measurement of outer and inner cabin and vehicle as well as the height above ground level. These variables have a direct impact on the driver's body postures and indirect one from the constant will of the driver to achieve best clear field-of view.

Task(s) Performance inputs. Understanding what and how is to be performed by the driver, including all the necessary variables (equipment needed, actions required, and time durations of the task). In case of a complex task, the task should be divided into task-stages that should all be studied separately as describes above. These variables will have later impact on the driver's body postures when using the various controls in order to complete the given tasks. It will also help to study the driver's clear field-of view.

Terrain and environment inputs. Understanding the various objective conditions in which the $\operatorname{task}(\mathrm{s})$ has to be completed, (e.g. terrain type: mud/ sand/rock, climate conditions: sun/rain/snow/fog, topography: plane/hill). These variables will have later impact on the driver's clear field-of view and body postures.

Ergonomic considerations, composed of visual, anthropometric and biomechanical analyses, and should be carried out in order to determine the degree of working comfort (visual and postural) of the driver. The results should also help to evaluate how well the cabin design fits the driver's anthropometry. The studies would be done on three different drivers' body percentiles (5\% ile, 50\%ile and $95 \%$ ile). The fundamental assumption is that these three percentiles can well represent most of driver's population that occupy the cabin. The studies are further detailed below:

\subsection{Visual Aspects in Multi-Task Vehicle Design}

Vision capabilities are crucial for vehicle driving tasks and maneuvers. When driving, the primary and most important information collecting sense modality is visual. The driver's visual sense is important for receiving information regarding both eye field-of-view, motion perception, and other inputs that are all accompanied by eye and head movements. It is even more complicated in a multi-task vehicle, as it presents the need for simultaneous visual monitoring. This is due to the fact that the actual driving, or steering actions, is always accompanied with other control action or actions. These additional actions vary, and can include controlling a plow dragged at back in case of an agricultural tractor; watching the cutters at the front in case of a combine; avoiding getting hit by bullets or shrapnel rain in case of an armored military vehicle, or, operating communication or weapon systems in case of military or emergency vehicles. All these scenarios share a common thing; they all demand additional visual attention followed by manual operations in addition to the ones needed for just driving the vehicle.

Vision requirements for vehicles are defined by regulations [26, 27, 28]. Forward field-of view is discussed in the 
EEC Directive 77/649. This defines the nearest visible areas in the front of the vehicle (downward and upward), and the obstruction of the side pillars of the windshield (A-pillars) all taking into account the accompanied eyes and head movement (turn).

Off road vehicle are still lacking existing vision standards as mentioned by [15], and may endanger the drivers that drive these vehicles because of many 'dead' or blocked areas that restrict the field-of view. These 'dead' areas are a direct result of the vehicle geometry, sometimes derived from faulty vehicle design.

Field-of-view mapping methods have been used for many years. The purpose of this mapping is to evaluate the actual field-of view, the human driver experience in vehicles. In 2000 [17] used a 'light bulb shadow test'. Disadvantage of this method are that the accuracy can be subjective and lacks repeatability. [2]Presented a new light bulb method based on light sensors. The mapping results were similar to the previous traditional method with an addition. The new technique presented a new ability of quantifying the data that was collected, and thus presented a clearer map of all blocked areas. As yet, the method is not entirely objective, as it uses a static light source that does not imitate exactly the human body with the capability of neck and head movements.

We suggest two different and interdepended studies in order to find out quantitatively how clear the driver's field-of-view actually is. These studies should be carried out for every task that has appeared above.

The first study is a spherical field-of-view tests that defines the maximum surrounding field-of-view that the operator can view around him using only fovea and peripheral eye sight, and with normal head and neck movement. It is important that the driver do not change his sitting posture in order to increase his clear field-of view. The view is done by binocular eye sight (Angel of 120 degrees, using both eyes).

Figure 1 presents example images of a computerized Spherical field-of-view study. The study is based on computerized simulations, with the use of commercial CAD programs used for mechanical engineering design and special ergonomic programs. Such programs are CATIA by DassaultSystemes, ManneQuinPRO by NexGen Ergonomics, and 3D SSPP program that is developed by the University Of Michigan College Of Engineering. The simulations include a digital model of the vehicle and the cabin with a digital-human-model (DHM) placed inside mimicking the exact body posture of a human driver. The simulation process includes s a 900 millimeter radius sphere, which circumscribes the vehicle with its center positioned at the operator's eye point. The sphere is made of a 5 degrees wire mesh that is positioned so its center is in the middle between the DHM's eye points. The 360/0 degree point is located in front or the driver; the 90 degrees point is at the right hand side of the driver, and so on.

The image on the bottom shows the program's output of the eye view of the DHM at a specific body posture. Here, the output presented is calculated by ManneQuinPRO program. By counting the visible intersections of the mesh's edges, the visible field-of-view can be calculated.

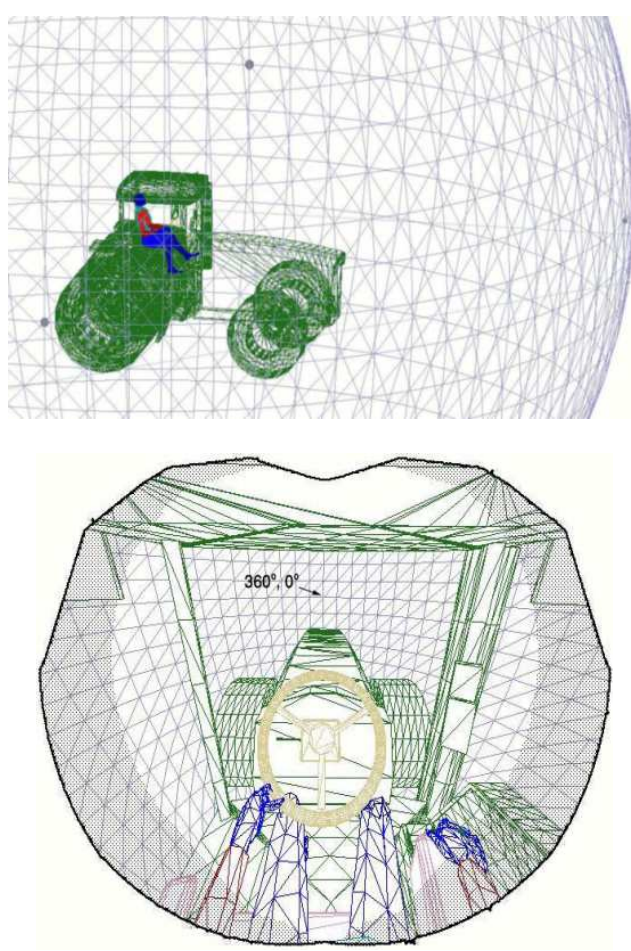

Figure 1. Spherical FoV analysis (Above: A distant view of a vehicle and the wire mesh sphere; Below: a DHM eye view.).

The other visual study consists on horizontally sliced field-of-view tests. This will test the clear field-of-view of a DHM at three height levels above ground: 50, 150 and 300 $\mathrm{cm}$. These heights are chosen as they represent the height of a child standing or a man kneeling in the proximity of the vehicle, a man standing upright, and a rooftop or a traffic light.

\subsection{Anthropometric Study of the Cabin Workplace}

In order to carry out the various given tasks, the driver operates the vehicle controls in a sedentary posture. Controls such as hand-controls and pedals can be described in terms of human outputs in order to control the vehicle. The hands are widely used for steering controls and various lever controls such as gear and hand-brake controls. The hands are also used to manipulate other controls such as joystick controls (found in tractors) and delicate switch controls. The feet are used mostly to provide outputs for longitudinal vehicle control such as acceleration or brake.

This sedentary posture has been acknowledged throughout the professional literature as problematic, and as a major risk factor and a contributor in the development of musculoskeletal disorders as suggested by ( $\mathrm{Li}$ and Haslegrave, 1999). This posture needs special attention during the design process, especially when accompanied by other manual manipulations that involve the use of force. The additional operations often cause adopting inadequate, un- 
comforted or 'bad' body postures, resulting in high risk of musculoskeletal disorder or even physical injury. (Sporrong et al., 1998) showed in their research that low static loads on the shoulders, that are a direct result of hand manipulation, can cause fatigue.

Many researches were conducted regarding the agricultural seat position and angles. Back in 1978, [3] have found that swiveling the tractor seat horizontally, improves the driver's sitting posture. Their results showed a decrease in the shoulders and back muscles activity (Trapezius and LatissimusDorsi). However, visual implications, as a result of this action, were not addressed in their research results. Today, most new manufactured tractors come with a 20 degrees swiveling option. Another research, conducted by [19] examined the effect of the seat backrest inclination with the transmission of vertical vibrations. Tests Results showed that the backrest inclination had impact on unloading various regions of the spine, especially the lumbar part. However, it had only a minor effect on the attenuation of the vibrations.

Some attempts were made to map the dimensions and location of the controls in field-vehicles. Several researches dealt with the manual operation of controls such as the research of [8]. They checked the location of controls against anthropometrical data. Other example is the research of [16] that found that the SAE 1994 standard for tractors, does not meet anthropometric requirements for manual hand reach. Other studies, such as the study of [24], checked the best location of the pedals in a tractor, in relation to the human strength needed for their operation. Another example is the research of [23] that checked the importance of adjustable pedals in private vehicles from the safety and comfort aspects. Their conclusion was that by maintaining the foot heel on the floor pan during braking or accelerating without the need of raising the foot, foot-ankle injuries are minimized, especially during a crash.

The anthropometric study suggests an evaluation of all physical elements inside the cabin. This evaluation checks the geometric location, dimension, angel, and position of every element, in relation to one another and in relation to the Seat Reference Point (SRP). In addition, the study evaluates the geometric location of all controls (foot and hand), and displays, inside the cabin, to best fit the operators' anthropometry. It also evaluates the controls for their location with the use of force.

\subsection{Biomechanical study}

During the years, epidemiological studies have shown connections between off-road driving and the association of increased risk of musculoskeletal disorders in the upper body and back. The importance of workplace dimensions and the interaction between the environment and the human factor was approached by [12]. They showed that awkward and uncomfortable body postures are adopted as a result of the workspace layout and dimensions. Moreover, [15] suggests that the sitting position of the driver and the 'workplace' design, have a huge impact on what the human driver is actually able to view, as these factors have a direct effect on the field-of view perception of the driver.

This study evaluates the loads on the driver's upper-body (neck, left and right shoulder, and lower-back), as a result of his sitting posture. The sitting posture is a direct result of the driver need to acquire manual reach distance for using of the controls and to obtain good vision on the road. The results outcome from this study will give the researcher the necessary information of the degree of comfort or the exposure to a potential risk, the driver feels when positioned inside the cabin.

\subsection{Summary and Conclusion: Verification and Validation}

All three studies results are to be analyzed by two different tools, especially designed for this purpose and are known to be accurate. A slight difference in the results $(0.05 \%)$ between the two tools is accepted, since every program uses different calculation algorithms. Results with higher difference, will suggest an error in the simulation, and the results will not be taken into account. In addition, comparing the studies' results with results deriving from a 'best fit ergonomic design cabin' is suggested. Since there is no real 'best fit' workplace, a workplace based on a collection of ergonomic rules and recommendations, taken from the scientific literature should be simulated. The comparison results can assist the verification of the correctness of the results and the degree of correlation to the real world.

A new improved cabin design will be accordingly suggested based on the results outputted. Later on, the discussed studies will be carried out on this new model suggested, the same three studies as before (e.g. vision, anthropometric, and biomechanical). Improvements in the improved model studies' results may validate the results.

\section{Results (Test-case Studies)}

\subsection{Introduction}

In order to test and evaluate the potential of the suggested ergonomic study, a multi-task off-road vehicle is examined as a test-case. The vehicle that is chosen is an agricultural tractor made by a well-known manufacturer, and is considered to be of top design. Two tasks are chosen for evaluation: driving forward on a road and plowing a field with a towed plow. These tasks involve more than one operation simultaneously, (e.g. steering the wheel while driving forward and looking backwards on the plow).

A computer model of the tractor is built from the manufacturer's blueprints. Using the ergonomic capabilities of the computer programs that were mentioned above, a DHM is placed inside the cabin in the proper sitting position. The various studies on the test case yields quantitative results as detailed below.

\subsection{Vision Studies and Results}

The vision studies on the test-case model, described above, 
yield the percentage of the clear field-of-view both for spherical and sliced studies. The results are distributed by three anthropometric DHM percentiles, type of task performed, field-of-view sectors, and by vertical height levels.

An example of the results is presented graphically in Figure 2. This example describes the results from a spherical field-of-view study, processed in MS-Excel spreadsheet. In this task the driver needs to have a constant eye view of the plow at the rear, together with a frontal eye view of the road ahead while driving. As a result, the seat in the tractor is swiveled 20 degrees to the right. By counting the wire mesh's intersections and documenting this data using a spreadsheet such as MS-Excel, quantitative date is gathered and mapped.

\begin{tabular}{|c|c|c|c|c|c|c|c|c|c|c|c|c|c|c|c|c|c|c|c|c|c|c|c|c|c|c|c|c|c|c|c|}
\hline & 360 & 5 & 10 & 15 & 20 & 25 & 30 & 35 & 40 & 45 & 50 & 55 & 60 & 65 & 70 & 75 & 80 & 85 & 90 & 95 & 100 & 105 & 110 & 115 & 120 & 125 & 130 & 135 & 140 & 145 & 150 \\
\hline 10 & & & & & & & & & & & & & & 10 & 10 & 10 & 10 & 10 & 10 & 10 & 10 & 10 & 10 & 10 & 10 & 10 & & 10 & & & \\
\hline 5 & 5 & 5 & 5 & 5 & 5 & 5 & 5 & 5 & 5 & & & & & 5 & 5 & 5 & 5 & 5 & 5 & 5 & 5 & 5 & 5 & 5 & 5 & 5 & & 5 & 5 & & \\
\hline 0 & 0 & 0 & 0 & 0 & 0 & 0 & 0 & 0 & 0 & & & & & 0 & 0 & 0 & 0 & 0 & 0 & 0 & 0 & 0 & 0 & 0 & 0 & 0 & & 0 & 0 & & \\
\hline-5 & -5 & -5 & -5 & -5 & -5 & -5 & -5 & -5 & -5 & & & & -5 & -5 & -5 & -5 & -5 & -5 & -5 & -5 & -5 & -5 & -5 & -5 & -5 & -5 & & -5 & -5 & & \\
\hline-10 & -10 & -10 & -10 & -10 & -10 & -10 & -10 & -10 & -10 & & & & -10 & -10 & -10 & -10 & -10 & -10 & -10 & -10 & -10 & -10 & -10 & -10 & -10 & -10 & & -10 & -10 & -10 & \\
\hline-15 & -15 & -15 & -15 & -15 & -15 & -15 & -15 & -15 & -15 & & & & -15 & -15 & -15 & -15 & -15 & -15 & -15 & -15 & -15 & -15 & -15 & -15 & -15 & -15 & & -15 & -15 & -15 & \\
\hline-20 & & & -20 & -20 & -20 & -20 & -20 & -20 & -20 & & & -20 & -20 & -20 & -20 & -20 & -20 & -20 & -20 & -20 & -20 & -20 & -20 & -20 & -20 & & & -20 & -20 & -20 & \\
\hline-25 & & & & -25 & -25 & -25 & -25 & -25 & & & & -25 & -25 & -25 & -25 & -25 & -25 & -25 & -25 & -25 & -25 & -25 & -25 & -25 & -25 & & & -25 & -25 & -25 & \\
\hline-30 & & & & & & & & & & & & -30 & -30 & -30 & -30 & -30 & -30 & -30 & -30 & -30 & -30 & -30 & & & & & & -30 & -30 & -30 & \\
\hline-35 & & & & & & & & & & & & -35 & -35 & -35 & -35 & -35 & -35 & -35 & -35 & -35 & -35 & & & & & & & & -35 & -35 & \\
\hline-40 & & & & & & & & & & & & -40 & -40 & -40 & -40 & -40 & -40 & -40 & -40 & -40 & & & & & & & & & & -40 & \\
\hline-45 & & & & & & & & & & & & -45 & -45 & -45 & -45 & -45 & -45 & -45 & -45 & & & & & & & & & & & -45 & \\
\hline-50 & & & & & & & & & & & & & -50 & -50 & -50 & -50 & -50 & -50 & & & & & & & & & & & & & \\
\hline-55 & & & & & & & & & & & & & & -11 & -11 & -11 & -11 & -11 & & & & & & & & & & & & & \\
\hline-60 & & & & & & & & & & & & & & & -12 & -12 & -12 & -12 & & & & & & & & & & & & & \\
\hline
\end{tabular}

Figure 2. Field-of-view mapping example of a plow task (50\%ile driver).

The cells that are visible are marked by a number, which presents the actual vertical angle above and below the horizon, (e.g. -5 means 5 degrees below the horizon). These cells are visible intersections on the sphere's wire mesh. Blank cells, without a number, are blocked intersections either by the vehicle's body, the wheels, side mirrors or A-pillars.

With the use of MS-Excel, histograms are generated based on the results, as seen in the example in Figure 3. The colored cells in this histogram represent clear view, while empty cells represent blocked view. Each vertical height level above or below the horizon is color coded. Each histogram shows the clear field-of-view map for a specific DHM percentile, distributed by vertical height level. Analyzing the histograms, the blocked cells in the front, result from the side mirrors and A-pillars that block the clear view of the driver. The blocked cells below the horizon level, result from the engine cover of the tractor and front wheels. In addition, the histograms show a 120 degree field-of-view sector defined by two black rays. This represents an actual eye field-of-view with the driver's head in natural position (i.e. no head rotation).

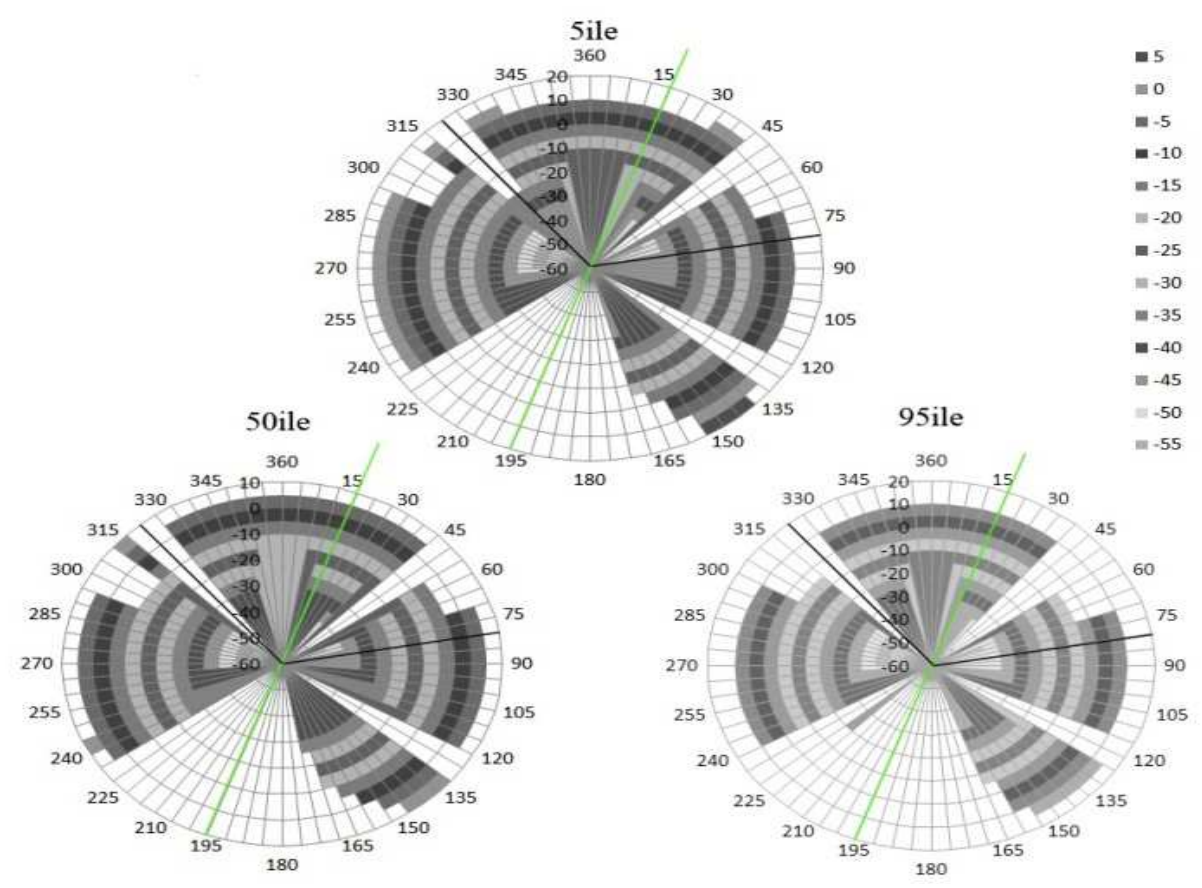

Figure 3. Field-of-view study results (5, 50 and 95 percentile comparison). 
One conclusion from these results can be that a 20 degrees seat rotation is not enough. In order for the driver to look at the rear of the vehicle, the driver has to adopt unnatural sitting positions.

The horizontally sliced field-of-view study consists also on a computerized model of a field vehicle's cabin settings with a DHM positioned inside. Figure 4 shows an example of such a simulation. In this example the vehicle is surrounded by a disk, located $150 \mathrm{~cm}$. above ground level. The disk's center aligns accordingly with the middle of the DHM's eyes position. Small round spheres are located at the outer rim of the disk, every 45 degrees, to serve as reference points. The disk is a 900 millimeter in radius, made of a 30 $\mathrm{cm}$. rings and 5 degrees sectors, wire mesh. Using the ergonomic add-on tool, through 'eye view' mode, it is easy identify and count the intersections and map the DHM's clear and blocked field-of-view. By counting the visible intersections of the sectors and rings, the visible field-of-view can be mapped and calculated. The data is later documented using a spreadsheet such as MS-Excel to produce quantitative information as was shown in the previous example.
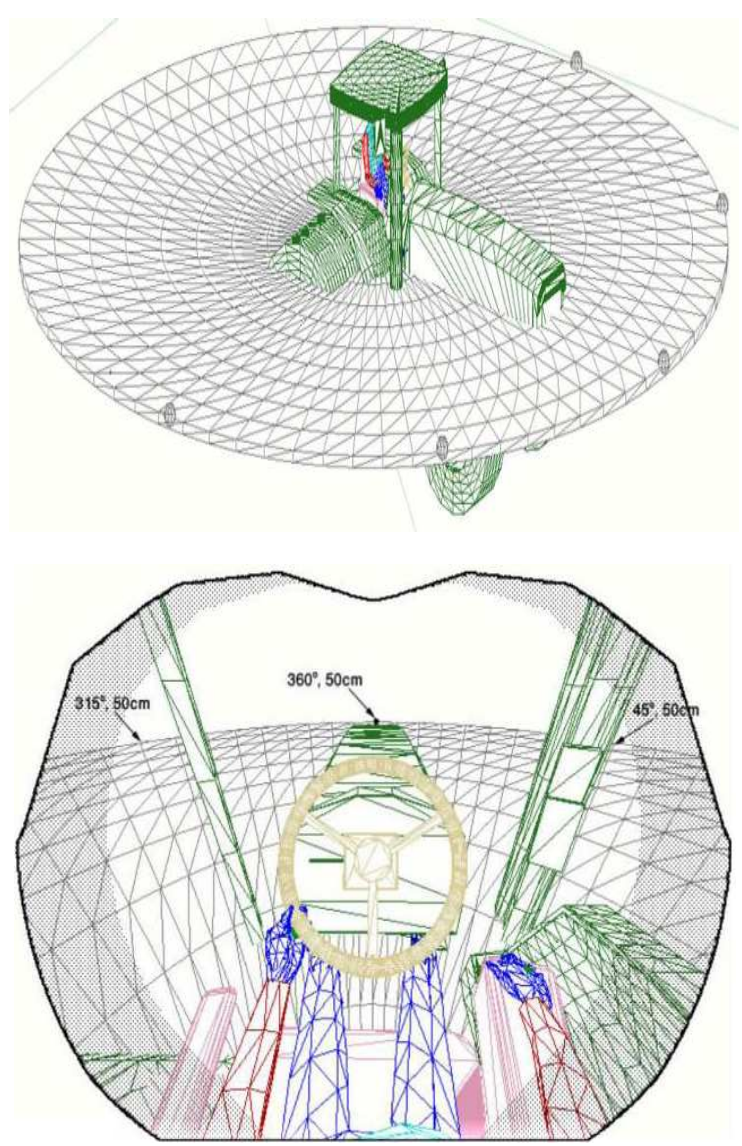

Figure 4. Horizontally sliced field-of-view analysis (Above: A distant view of a vehicle and the wire mesh disk at $150 \mathrm{~cm}$. above ground level; Below: A DHM eye view.).

This study is done three times. Each time, the disk is placed is another height level, with the center at the middle point of the DHM's eye points.

\subsection{Anthropometric Studies}

The anthropometric results generated from the test-case analysis, show graphically the driver's manual and foot reach envelopes for each DHM percentile. The study is performed only after the driver reaches sitting posture that allows him the best clear field-of-view, for the task given at that point. The study results are distributed by three anthropometric percentiles and type of task performed. By tagging every control in the cabin, quantitative information regarding reach and operational capabilities can be obtained.

Figure 5, shows an example of anthropometric study using a computer simulation of a cabin layout with a DHM positioned inside. The DHM is positioned imitating a real human driver, operating the vehicles controls. By generating hand and foot reach envelopes (performed in this case using ManneQuinPRO program and appears in wire mesh), helps to inspect the controls' location, and can help identify if any are out of hand or foot reach, which may affect the natural sitting posture.
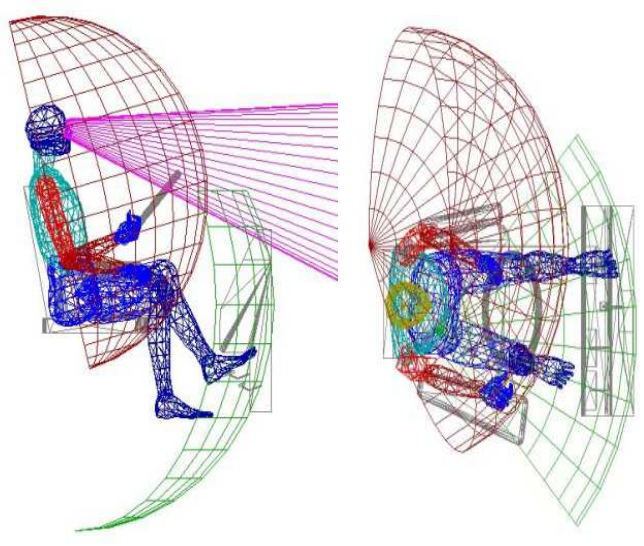

Figure 5. Anthropometric simulations analysis. Depicted 3D mesh represents maximum hand reach envelopes. Same can be generated for the feet.(Left: Side view. Right: top view).

\subsection{Biomechanical Studies}

The biomechanical study results generated from the test-case yield the loads on the human upper body as a result of the body posture that is adopted. The study is performed only after the driver reaches sitting posture that allows him best clear field-of-view, and best manual and foot control-handling, for the task given at that point. The results are distributed by three anthropometric percentiles and type of task performed.

The example presented in Figure 6 shows a histogram output from biomechanical computer simulations on three different DHM body percentiles. In this example the simulations are tested using RAMSIS and 3D SSPP programs. The results are a comparison of upper body loads values divided into three anthropometric percentiles of DHMs, generated by MS-Excel program. 


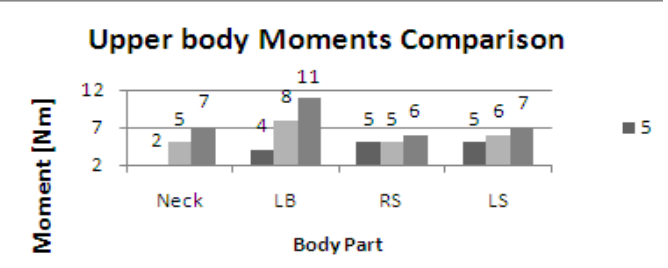

Figure 6. Biomechanical simulations analysis (5, 50 and 95 percentile DHM comparison).

In addition, different backrest inclinations are tested in order to minimize the overall upper body loads. The main idea is to find the best tradeoff between the different body parts (e.g. increase of back rest inclination and extending of the arm and hand). The results show that the best trade-off inclination angel for the backrest of the seat in the specific sitting posture is 103-104 degrees. The example in Figure 7 shows biomechanical study of a $50 \%$ ile driver positioned in driving task inside the test-case tractor. The driver is positioned in a sitting position that obtains best clear field-of-view, and all controls' are in its reach envelopes, all correlates to the specific task given. Upper body loads (neck, right and left shoulders and lower back) are then studied.

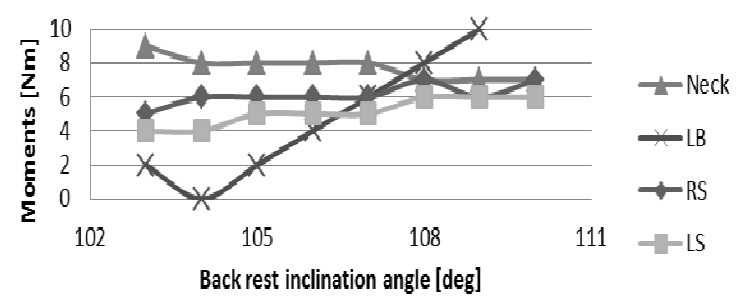

\subsection{Summary and Conclusion}

The various results, which are gathered from all three studies on the test-case, yield quantitative and unequivocal results. Based on these results a better task-performance cabin design can be accomplished. This new design will follow ergonomic rules and guidelines for the safety of the driver in mind for more visible visual field-of-view, better sitting postures, and easy access and handling of the controls through a better layout, all lead to smaller biomechanical loads on the operator's upper body.

Figure 8 shows a skeleton flowchart of the studies we suggest. After first inquiries about the type of vehicle, task and terrain, three different ergonomic studies are performed on a chosen multi-task vehicle (A). A chosen multi-task vehicle is studied on three separate studies as described earlier. Same studies are performed on a vehicle model that is generated from scratch based on scientific literature that includes ergonomic guidelines and recommendations (B). This process is done in order to verify the results outputted from the studies on vehicle (A).

Based on the results gathered from the studies on vehicle (A), a new and improved vehicle model can be suggested (C). This new vehicle model will be studied and the results will be compared to the results outputted from vehicle (A). Improvement in the result outputted from the new and improved vehicle will show a success and will also validate the studies' results.

As mentioned before, the design of the new and improved vehicle (C), will follow ergonomic guidelines, thus will reduce the need of unnatural body postures and will increase the clear field-of-view of the driver. As an outcome this new vehicle will be safer for use for the human driver.

Figure 7. Biomechanical study results.

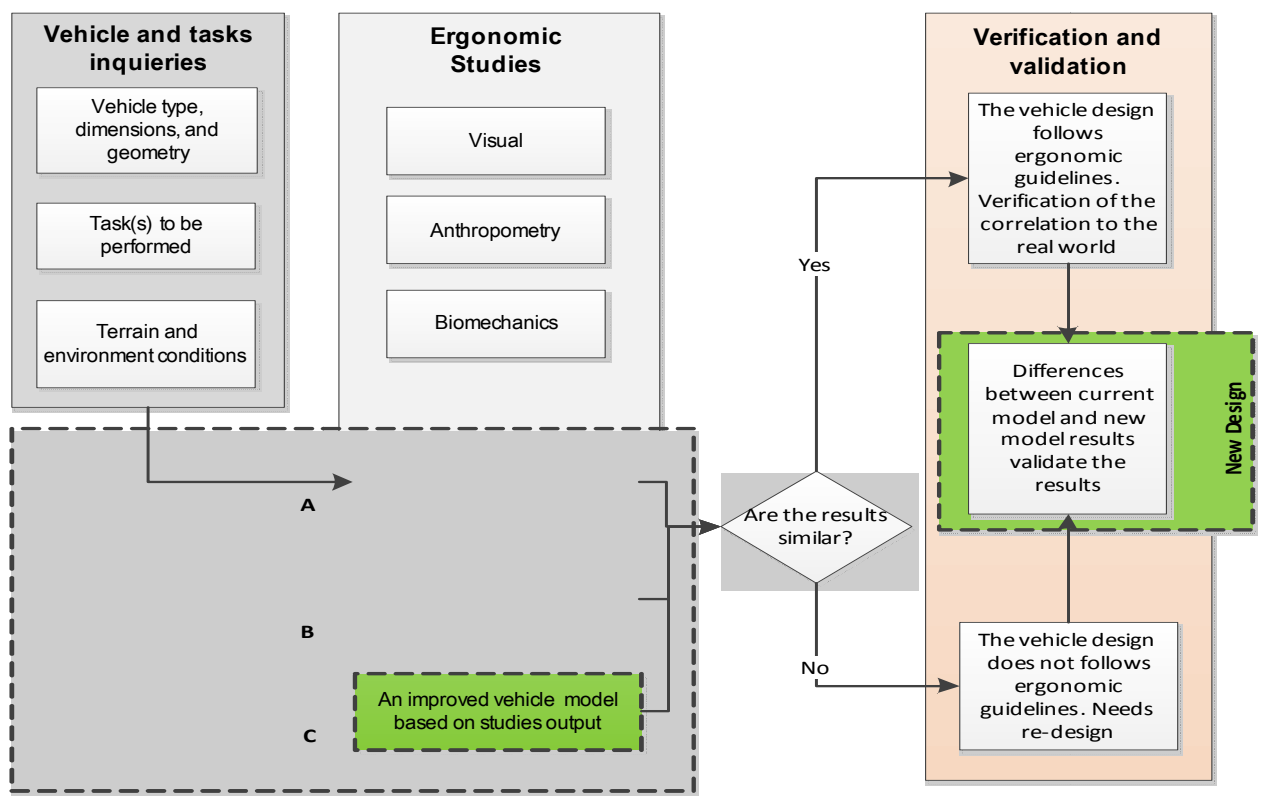

Figure 8. Flowchart of the ergonomic studies process. 


\section{Discussion}

A new experimental ergonomic study is presented here to tackle the difficult and challenging cabin design of a multi-task off-road vehicle. A vehicle, as explained before, has a very complicated working environment, from the ergonomic point-of-view. Here, a new approach tries to manage the compromises necessary to achieve an overall acceptable design, based on three ergonomic studies. All three studies are combined together and depend on one another, in order to increase the driver's ergonomic effectiveness. The literature search, did not present such an ergonomic design process that takes in count vision, anthropometric and biomechanical aspects all at the same time.

Designing the cabin geometry and controls for best fitting to the human dimensions and manual and foot capabilities is not enough, as some researches have suggested. In order to present a better ergonomic design one must take in account the visual aspect as well, as it is also derived from the human sitting posture in the cabin. Once the controls are positioned around the driver in a way that minimizes the need to sit in unnatural postures, accompanied with a clear field-of-view with minimum blocked areas, one provides a better task-performance design. This better design enables the driver to drive in comfort, in a more vigilant way and with a good view on the road ahead. All these, may minimize fatigue and road accidents that are deriving out of human errors.

\section{References}

[1] Attaianese, E. and Duca, G., (2011). Human factors and ergonomic principles in building design for life and work activities: an applied methodology. LEAS, Faculty of $\mathrm{Ar}$ chitecture, University of Naples Federico II. Firenze University Press. pp. 118-127.

[2] Barron, P., J., Owende, P., M., O., McDonnell, K., P., Ward, S., M., (2005). A Method for Assessment of Degradation of Task Visibility from Operator Cabins of Field Machines.International Journal of Industrial Ergonomics, 35(7). pp. $665-673$.

[3] Bottoms, D.J., barber, T.S., (1978). A Swiveling Seat to Improve Tractor Driver's Posture. Applied Ergonomics, 9(2). pp. $77-84$.

[4] Brown, S. (1985). Ergonomics in design education: a user-centered approach. Design Studies, 6 (2), pp 73-82.

[5] Chaffin, D.B., (2007). Human Motion Simulation for Vehicle and Workplace Design. Human Factors and Ergonomics in Manufacturing, 17 (5). pp. 475-484.

[6] Charytonowicz, J., (2000). Architecture and Ergonomics. Human Factors and Ergonomics Society Annual Meeting Proceedings, Proceedings 6 - General Sessions , pp. 103-106(4).

[7] Corlett, E., N., (1983). Analysis and evaluation of working posture, in T. O. Kvålseth (ed). Ergonomics of Workstation Design. Butterworth-Heinemann. pp. 1-18.

[8] Drakopoulos, D., Mann, D., D., (2006). An Ergonomic Analysis of the Controls Present in a Tractor Workstation. Presented at the Canadian Society for Bioengineering, 2006 Annual Conference, Edmonton Alberta, July 16-19.

[9] EEC, council Directive 77/649 (1977). Field of vision of motor vehicle drivers. Official Journal of Europian Communities, L267. Brussels. pp.1 -22.

[10] European ergonomic and safety guidelines for forest machines, (2006). Gellerstedt S., Eriksson, G., Frisk. S., Hultåker, O., Synwoldt, U., Tobisch, R., Weise, G., (Eds.). A handbook produced by ErgoWood, a project co-financed by the European Commission.

[11] Franklin, R., Mitchell, R., Driscoll, T., Fragar, L., 1999. Farm-Related Fatalities in Australia, 1989-1992. Australian Centre for Agricultural Health and Safety and Rural Industries Research and Development Corporation, pp. 213-225.

[12] Gilad, I., Byran, E., (2007). Ergonomic evaluation of the ambulance interior to reduce paramedic discomfort and posture stress, Human Factors, 49(6).

[13] Guangyan, L., and Haslegrave, C., M., (1999). Seated work for manual, visual and combined tasks. Ergonomics, 42 (8). pp. 1060-1086.

[14] Hasdoğan, G.,(1996). The role of user models in product design for assessment of user needs. Design Studies, 17 (1), pp 19-33.

[15] Haslegrave, C., M., (1993). Visual aspects in Vehicle design. Automotive Ergonomics, Edited by Peacock, B. and Karwowski, W.Taylor and Francis Ltd. pp. 43-79.

[16] Hsiao, H., Whitestone, J., Bradtmiller, B., Whisler, R., Zwiener, J., Lafferty, C., Kau, T., Y., and Gross, M., (2005). Anthropometric criteria for the design of tractor cabs and protection frames. Ergonomics, 48(4). pp. $323-353$.

[17] Hutter, G.,M., 2000. Earthmoving equipment - an operator's field of view (S. A. E standard J1091). Safety and Forensic Engineering Journal 2.

[18] Li, G., and Haslegrave, C., M., (1999). Seated work postures for manual, visual and combined tasks. Ergonomics, 42(8). Taylor and Francis. pp. 1060-1086.

[19] Magnusson, M., Pope, M., Rostedt, M., Hansson, T., (1993). Effect of Backrest Inclination on the Transmission of Vertical Vibrations through the Lumbar Spine. Clinical Biomechanics, 8(1), pp. 5-12.

[20] MIL-STD-1472F, (1999). Department of Defense Design Criteria Standard, Human Engineering. Distribution Statement A: Approved for public release; distribution is unlimited.

[21] Moody, S. (1981). Ergonomic principles in the design of hand tools: T M Fraser ILO, Geneva, Switzerland (1980).Design Studies, 2 (4). pp. 93.

[22] NASA-STD-3000, (1995). Man-System integration Standards. NASA, National Aeronautics and Space Administration. 
[23] Parenteau, C., S., Shen, W., Shah, M., (2000). The Effectiveness of Adjustable Pedals Usage. Reprinted from: Human Factors in 2000: Driving, Lighting, Seating Comfort, and Harmony in Vehicle Systems. SAE World Congress, Detroit Michigan. pp. 1-9.

[24] Pheasant, S., T., and Harris, C., M., (1982). Human strength in the operation of tractor pedals. Ergonomics in Agriculture and Forestry, 25(1). Taylor and Francis Ltd. pp. 53-64

[25] Rötting, M., Rösler, D., Lohse, K., Göbel, M., (2000). Activity and Eye Movement Analysis as Basis of Vehicle Cabin Design. Proceedings of the XIVth Triennial Congress of the IEA and 44th Annual Meeting of the HFES, San Diego, California, 3, pp. $341-344$.

[26] SAE, 1973a, SAE Recommended Practice J182a, Motor
Vehicle Fiducial marks, New York: Society of Automotive Engineers.

[27] SAE, 1973b, Field of view from automotive vehicles, SAE SP-381, New York: Socity of Automotive Engineers.

[28] SAE, 1994, Surface Vehicle Standard - Control Locations for Off-Road Work Machines, SAE J898 (Warrendale, PA: Society of Automotive Engineers, Inc).

[29] Schlegel, R., E., (1993). Driver mental workload. Automotive Ergonomics, Edited by Peacock, B. and Karwowski, W.Taylor and Francis Ltd. Pp. 359-383.

[30] Sporrong, H., Palmerud, G., Kadefors, R., Herberts, P., (1998). The effect of light manual precision work on shoulder muscles-an EMG analysis. Journal of Electromyography and Kinesiology, 8. Pp. 177-184. 